\title{
MD Simulation of AEDG Peptide Complexes with New K2R Dendrimer and Dendrigraft
}

\author{
E.I. Fatullaev, V.V.Bezrodnyi, I.M. Neelov \\ ITMO University, Kronverkskiy pr. 49, St. Petersburg, 197101, Russia
}

Received: June 26, 2021. Revised: November 10, 2021. Accepted: December 13, 2021. Published: January 3, 2022.

\begin{abstract}
Biocompatible peptide dendrimers and dendrigrafts have useful properties for application in biomedicine. In previous papers the computational approach for study lysine dendrimers and dendrigrafts as well as their complexes with various medical peptides was used. In this paper the comparison of complex formation between molecules of therapeutic AEDG tetrapeptide and novel K2R peptide dendrimer or DG2 dendrigraft of near the same size and charge was fulfilled. The systems consisting of 16 therapeutic AEDG tetrapeptide molecules and one dendrimer or one dendrigraft were studied by molecular dynamics simulation. Full atomic models of these molecules in water with explicit counterions were used for this goal. First of all, the process of complex formation was studied. It was obtained that peptide molecules were attracted by both branched molecules and were quickly adsorbed by them. Times of complexes formation as well as size, anisotropy and structure of each complex were calculated. It was demonstrated that both K2R dendrimer and DG2 dendrigraft are effective for complexation of these peptide molecules but new dendrimer complex is more stable than dendrigraft complex because it has almost twice more hydrogen bonds with peptide molecules and $33 \%$ more ion pairs with their charged groups.
\end{abstract}

Key-Words: - AEDG tetrapeptide, complex, K2R peptide dendrimer, lysine dendrigraft.

\section{INTRODUCTION}

It is well known that therapeutic peptide (AEDG) consisting of 4 aminoacid residues Ala-Glu-Asp-Gly has neuroprotective properties [1-3]. But since it has a negative charge, it is difficult for it to penetrate cell membranes that have a charge of the same sign. Elaboration of novel carriers for gene and drug delivery is important direction in pharmaceutics now. Highly branched molecules including dendrimers and dendrigrafts are good candidates for this goal. Lysine dendrimers [4] are well known regularly branched molecules with single branching center (core). Dendrigrafts are also highly branched molecules but they have several branching cores located on very short linear peptide chain. Lysine dendrigrafts are not as regular molecules as lysine dendrimers but their $3 \mathrm{~d}$ structure is similar with that of dendrimers. These branched molecules could be functionalized by other aminoacid residues (for example by arginine or histidines) or by short peptides or by other bioactive groups or molecules [5-8]. Usually, functionalization is carried out for the terminal groups of dendrimers and dendrigrafts [9], but here the functionalized of dendrimer volume was done. The pairs of arginine residues (2Arg) were inserted between all adjacent branch points of a conventional lysine (Lys (or $\mathrm{K}$ in one letter notation)) dendrimer resulting in a peptide dendrimer with a Lys2Arg (K2R in one letter notation) repeating branched unit.

Both lysine peptide dendrimers and lysine dendrigrafts have many positively charged groups. That's why they are suitable for use as antiviral and antibacterial drugs $[10,11]$ and in drug and gene delivery.

Main aim of our paper is to study complex formation and structure of complex made by K2R dendrimer or lysine dendrigraft with AEDG tetrapeptide molecules.

\section{METHOD of MOLECULAR DYNAMICS}

The method of molecular dynamics (MD) is widely used for study all biopolymer systems including proteins, peptides, DNA and RNA as well as different polysaccharides and lipid membranes. This method uses numerical solution of Newton equations for each atom of simulated system.

In this paper MD simulation was performed for systems containing lysine dendrigraft or lysine dendrimer and 16 AEDG peptides. Dendrigraft of second generation DG2 has charge +48 (48 charged NH3 + groups), K2R peptide dendrimer of similar molecular mass has repeatimg units K2R (lysine-2arginine) and charge +44 (+28 from internal arginine aminoacid residues and +16 from 16 lysine terminal groups). Each of 16 AEDG peptides has charge -2 (consisting of charge -1 of COO- side group of Glu (E) and Asp (D) aminoacid residues). Periodical cubic simulation cell filled by water with addition of counterions to keep system electroneutral was used. 


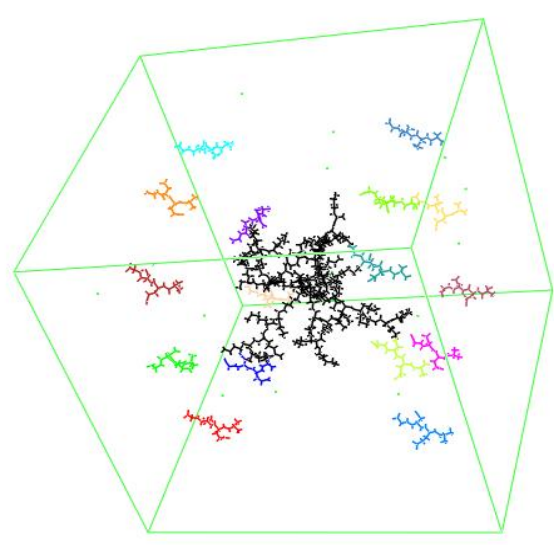

(a)

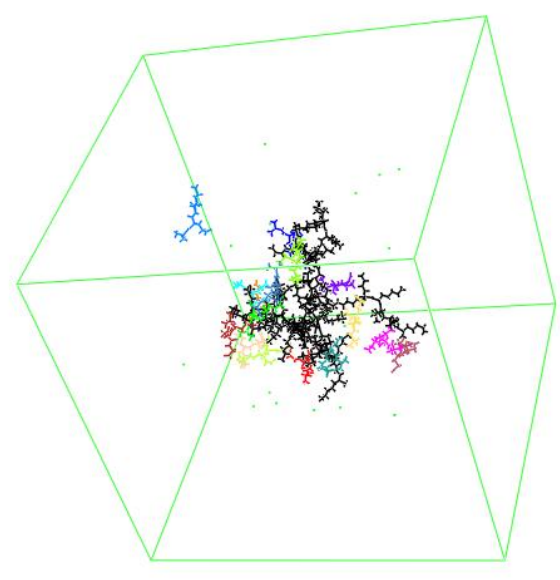

(b)

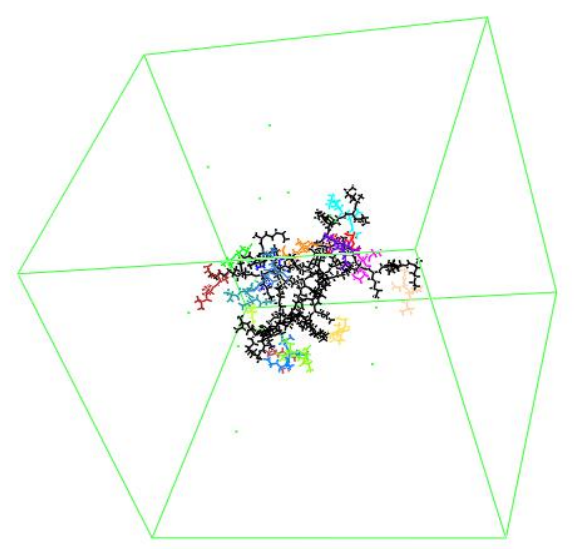

(c)

Figure 1. Snapshots of conformations at a) initial time $(\mathrm{t}=0)$ and after time $\mathrm{b}) \mathrm{t}=4 \mathrm{~ns}$ and $\mathrm{c}) \mathrm{t}=10 \mathrm{~ns}$ of simulation of lysine dendrigraft of generation $\mathrm{G}=2$ (DG2) and 16 AEDG tetrapeptides. Dendrigraft is marked by black color and tetrapeptides by different colors.

The initial conformations of lysine dendrigraft DG2 and $\mathrm{K} 2 \mathrm{R}$ dendrimer were chosen from our previous MD calculations of single DG2 or K2R branched molecule in water (no tetrapeptide molecules). For all tetrapeptide molecules the same initial conformation (dihedral angles corresponding to beta-sheet conformation of main peptide chain of all aminoacid residues) was prepared using molecular editors. Initial energy minimization and all MD

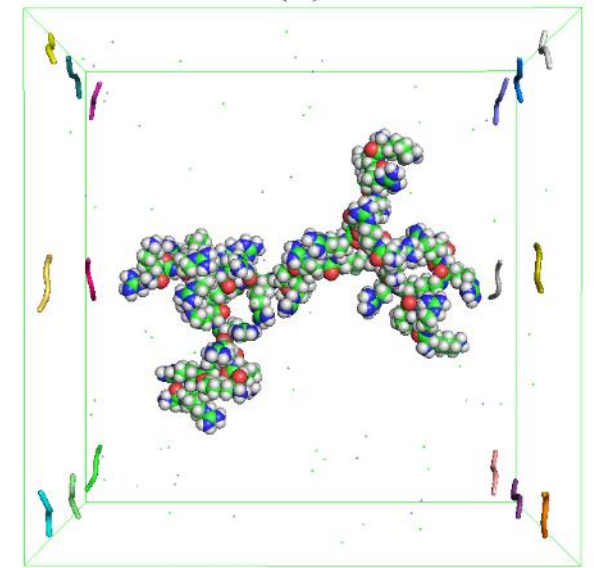

(a)

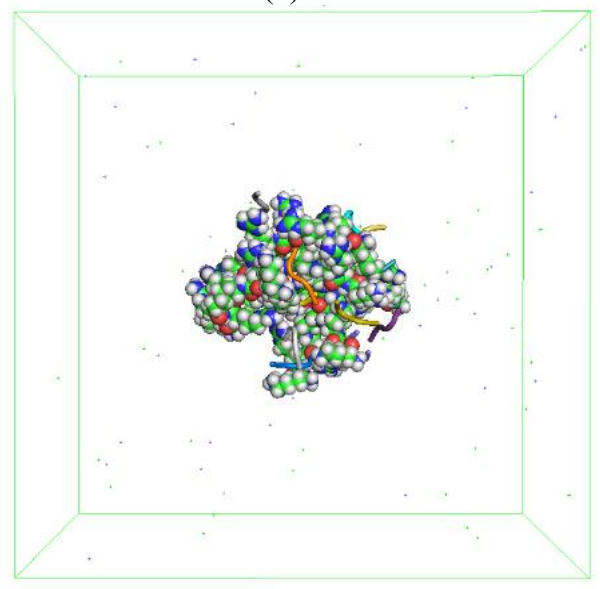

(b)

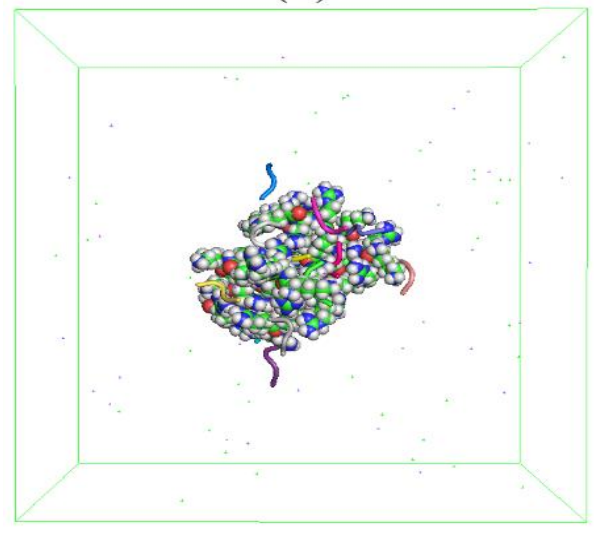

(c)

Figure 2. Snapshots of conformations at (a) initial time $(\mathrm{t}=0)$ and after time $\mathrm{b}) \mathrm{t}=10 \mathrm{~ns}$ and $\mathrm{c}) \mathrm{t}=25 \mathrm{~ns}$ of simulation of dendrimer of generation $\mathrm{G}=2(\mathrm{~K} 2 \mathrm{R})$ and 16 AEDG tetrapeptides. Dendrigraft atoms are shown as beads of different color.

calculations use GROMACS package [12] and AMBER99SB-ildn version of forcefields [13].

LINCS algorithm to constraint all valence bonds and particle mesh Ewald algorithm (PME) for correct calculation of electrostatic interactions in systems with periodical boundary conditions were used in all simulations. All calculations consisted of 100-200ns trajectories for both systems and have shown that 
equilibration in both of them occur very quickly. Thus, we divided full trajectory for two parts: initial part for complex formation and second part for calculation of equilibrium properties (size, shape and internal structure) of complex. In all MD calculations the NPT ensemble was used. Temperature was equal $300 \mathrm{~K}$ and pressure was equal 1 ATM.

The main details about MD sinulation of linear polyelectrolytes is described in [14, 15, 17]. Similar information about MD simulation of lysine dendrimers could be found in [39-40] and information about MD simulation of new peptide dendrimers could be found in papers [60-62].

Futher information on simulation of linear polymers and polyelectrolytes [14-35], branched and hyperbranched polymers [36-62] and complexes of branched molecules with peptides [63-70] have been described earlier and could be found there.

\section{COMPLEX FORMATION}

Snapshots of DG2 dendrigraft with tetrapeptide molecules as well as K2R dendrimer with the same tetrapeptide molecules at different time moments are shown on Fig. 1 and Fig.2, correspondingly (water and counterion molecules are not shown for clarity. It is clearly seen that at the beginning of simulation (Fig. 1a and Fig.2a) all peptide molecules are far from dendrigraft and dendrimer correspondingly. After short simulation time $(\mathrm{t}=4 \mathrm{~ns}$ in Fig. $1 \mathrm{~b}$ and $\mathrm{t}=10 \mathrm{~ns}$ in Fig. $2 \mathrm{~b}$ ) almost all molecules of tetrapeptide are adsorbed and after $t$ equal 10ns and 25ns correspondingly (Fig.1c and Fig. 2c) all tetrapeptide molecules are adsorbed completely.

To characterize the size of the subsystem consisting of DG2 dendrigraft or K2R dendrimer together with tetrapeptide molecules the instant square of radius of gyration $R g 2(t)$ was used. The value of instant radius of gyration $R g$ were calculated using g_gyrate function of GROMACS package.

The time dependences of $R g(t)$ for these subsystems describe the process of equilibration and demonstrates the kinetics of complex formation (if formation of complex occurs). It can be seen from Fig. 3a and Fig4a, that in the beginning of simulation $(t=0)$ the value of $R g$ is rather large for both subsystems. After that the radius of gyration of the subsystems become smaller and smaller because It is easy to see that at the beginning of simulation this function is equal 0 in both systems because tetrapeptide molecules are far from dendrigraft or dendrimer and due to this reason do not have contacts with them. When some tetrapeptide molecules become closer to dendrigraft or dendrimer the first contacts between them appears and number of hydrogen bonds increase with time. peptide molecules are attracted by dendrigraft and dendrimer and become more and more close to it due to strong intermolecular interactions. The slope of initial part of time dependence of $R g(t)$ where it decreases with time could characterize the rate of complex formation. After initial decrease, the value of $R g(t)$ fluctuates but its average value practically doesn't change with time. It means that $R g(t)$ goes to plateau value because all peptide molecules become adsorbed on dendrigraft surface. It occurs at time about $10 \mathrm{~ns}$ in Fig. $3 \mathrm{a}$ and $25 \mathrm{~ns}$ in Fig.4a correspondingly.

The time dependences of distance between K2R dendrimer or dendrigraft and peptide molecules (Fig.3b and Fig.4b, correspondingly) also demonstrates how quickly peptide molecules become adsorbed by dendrigraft and dendrimer. Function "distance" was calculated using $g \_$bond function of GROMACS package. Its behaviour is similar to that of radius of gyration (see previous plots Fig.3a and Fig.4a). The distances again are rather big at the beginning of simulation than they quickly decrease during first $10 \mathrm{~ns}$ and $25 \mathrm{~ns}$ correspondingly and after that go to plateau. Thus the time dependence of distance is similar with behavior of radius of gyration for each system. Due to this reason one can assume that after first $10 \mathrm{~ns}$ and $25 \mathrm{~ns}$ correspondingly both systems are close to equilibrium state and calculate equilibrium values of $R g=\operatorname{sqrt}\left(<R^{2}(t)>\quad\right.$ (where $<>$ mean average on equilibrium (plateau) and mean square distances between dendrigraft or dendrimer using second part of trajectory (for example, time $\mathrm{t}>30 \mathrm{~ns}$ ).

Another quantity that can demonstrates kinetic of complex formation and confirm that the dendrigraft or $\mathrm{K} 2 \mathrm{R}$ dendrimer adsorbs tetrapeptide molecules is the time dependence of number of hydrogen bonds between dendrigraft or dendrimer and peptide molecules. This characteristic was calculated using g_hbonds function of GROMACS package (Fig.3c and Fig.4c for dendrigraft-tetrapeptides and dendrimer- tetrapeptides, correspondingly).

Another quantity that can demonstrates kinetic of complex formation and confirm that the dendrigraft or K2R dendrimer adsorbs tetrapeptide molecules is the time dependence of number of hydrogen bonds between dendrigraft or dendrimer and peptide molecules. This characteristic was calculated using $g$ _hbonds function of GROMACS package (Fig.3c and Fig.4c for dendrigrafttetrapeptides and dendrimer- tetrapeptides, correspondingly). It is easy to see that at the beginning of simulation this function is equal 0 in both systems because tetrapeptide molecules are far from dendrigraft or dendrimer and due to this reason do not have contacts with them. When some tetrapeptide molecules become closer to dendrigraft or dendrimer the first contacts between them appears and number of hydrogen bonds increase with time. After time $\mathrm{t}$ become close to $10 \mathrm{~ns}$ for dendrigraft and about $25 \mathrm{~ns}$ for dendrimer this function goes to almost its maximum value for the first time 


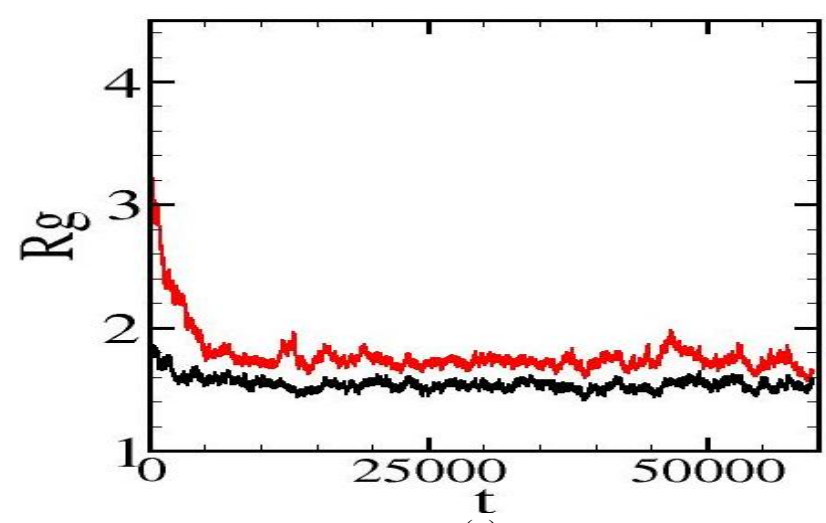

(a)

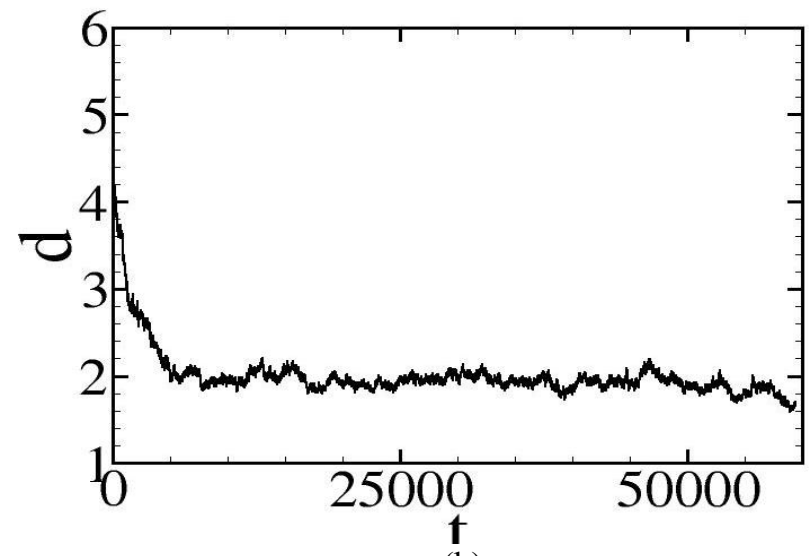

(b)

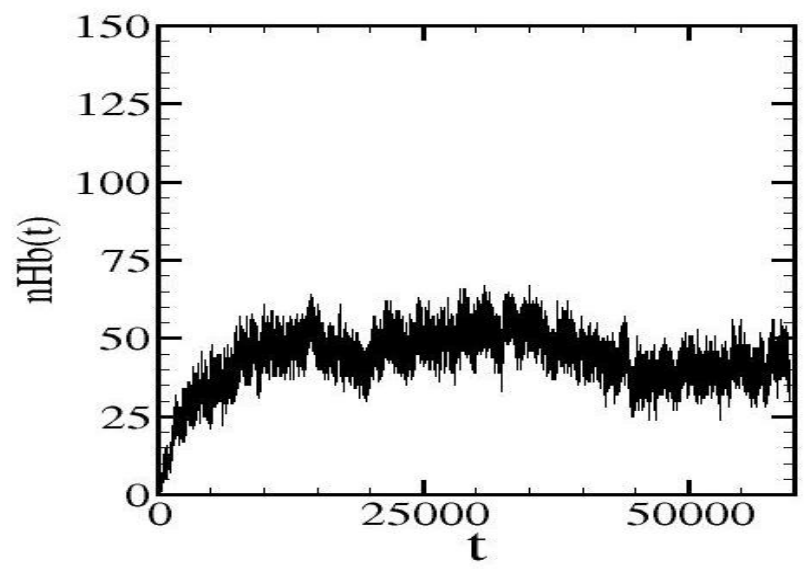

(c)

Figure 3. a) Time dependences of: a) gyration radius $R_{g}$ of dendrimer (black) \& complex (red) (b) distance $d$ and (c) number of H-bonds $(\mathrm{nHb})$ between DG2 dendrigraft and tetrapeptide molecules.

because all peptide molecules are already in contact with dendigraft. At larger times the number of hydrogen bonds fluctuates (due to rearrangement of tetrapeptide molecules on surface of dendrigraft/dendrimer) but its average values almost don't change with time. It means that these characteristics reached their plateau value for each system (near 50 and $100 \mathrm{H}$-bonds correspondingly) and become close to their equilibrium values.

\section{EQUILIBRIUM COMPLEX}

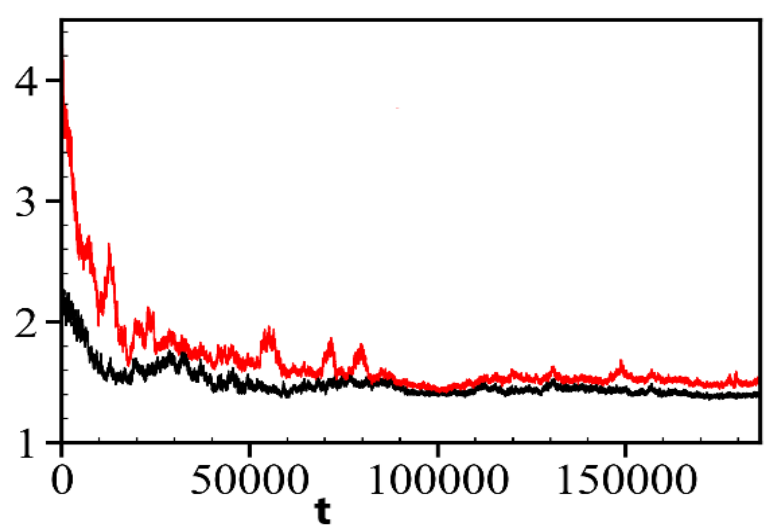

(a)

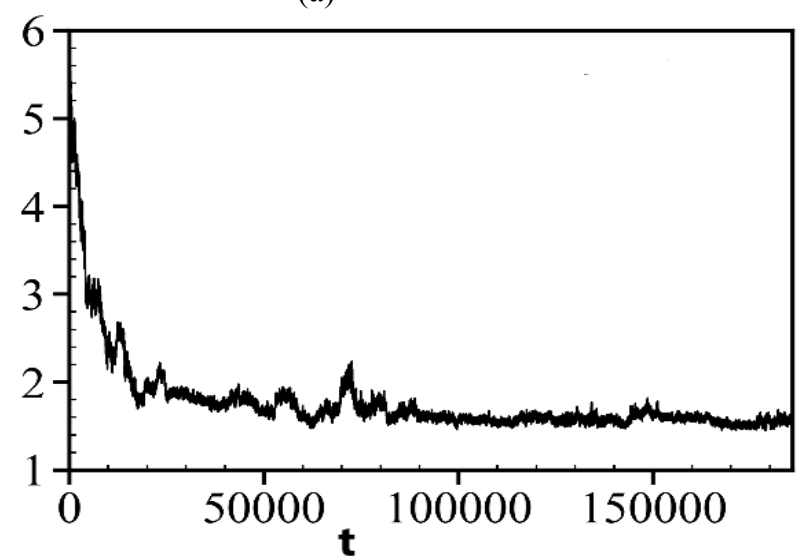

(b)

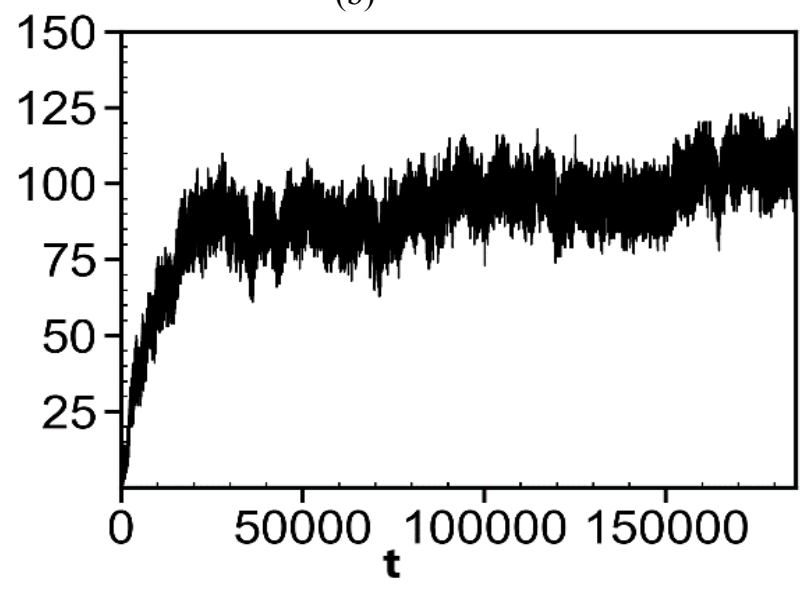

(c)

Figure 4 a) Time dependences of: a) gyration radius $R_{g}$ of dendrimer (black) \& complex (red), (b) distance $d$ and, (c) number of $\mathrm{H}$-bonds $(\mathrm{nHb})$ between $\mathrm{K} 2 \mathrm{R}$ dendrimer and tetrapeptide molecules.

Equilibrium values of $\mathrm{Rg}$ of $\mathrm{DG} 2$ dendrigraft or K2Rdendrimer and their components in complexes (see also Fig3a and Fig4a and more detailed information about $\mathrm{Rg}$ for similar dendrimer-peptide complexes [67-71]).

The shape anisotropy calculated as ratio $R^{33} / R^{11}$ of longest $R g^{33}$ and shortest $R g^{11}$ axes of equivalent ellipsoid of branched molecules and complexes as a whole are shown in table 1. The size of free DG2 dendrigraft and $\mathrm{R} 2 \mathrm{~K}$ dendrimer in water are equal $1.8 \mathrm{~nm}$ and $2.0 \mathrm{~nm}$ 
correspondingly. Thus, their sizes in complex are 1.18 and 1.40 times less in complexes with the same tetrapeptides than in free state in water. It means that dendrimer in complex is more compacted than dendrigraft.

Table 1. Values of $R_{g}{ }^{11}, R_{g}{ }^{22}, R_{g}{ }^{33}, R_{g}(\mathrm{~nm})$ and anisotropy for dendrigraft DG2 and its complex with $16 \mathrm{AEDG}$ and for dendrimer K2R and its complex with 16AEDG peptide molecules.

\begin{tabular}{lccccc} 
System & $\mathrm{R}_{\mathrm{g}}{ }^{11}$ & $\mathrm{R}_{\mathrm{g}}{ }^{22}$ & $\mathrm{R}_{\mathrm{g}}{ }^{33}$ & $\mathrm{R}_{\mathrm{g}}$ & $\mathrm{R}_{\mathrm{g}}{ }^{33} / \mathrm{R}_{\mathrm{g}}{ }^{11}$ \\
\hline $\mathrm{DG} 2$ & 1.05 & 1.29 & 1.38 & 1.53 & 1.31 \\
$\mathrm{DG} 2+16 A E D G$ & 1.21 & 1.44 & 1.57 & 1.73 & 1.30 \\
K2R & 0.91 & 1.23 & 1.30 & 1.42 & 1.43 \\
K2R+16AEDG & 1.00 & 1.29 & 1.39 & 1.51 & 1.39 \\
\hline
\end{tabular}

It is easy to see that the size of the complex of DG2 with 16 AEDG peptide molecules is 1,13 times larger than the size of the dendrigraft DG2 in the complex. At the same time the size of dendrimer-terapeptides complex is only 1,05 times greater than size of dendrimer. It means that peptide molecules are slightly closer to center of dendrimer than to center of dendrigraft. These result correlates also with the 1.15 greater size of complex with dendrigraft than complex with dendrimer.
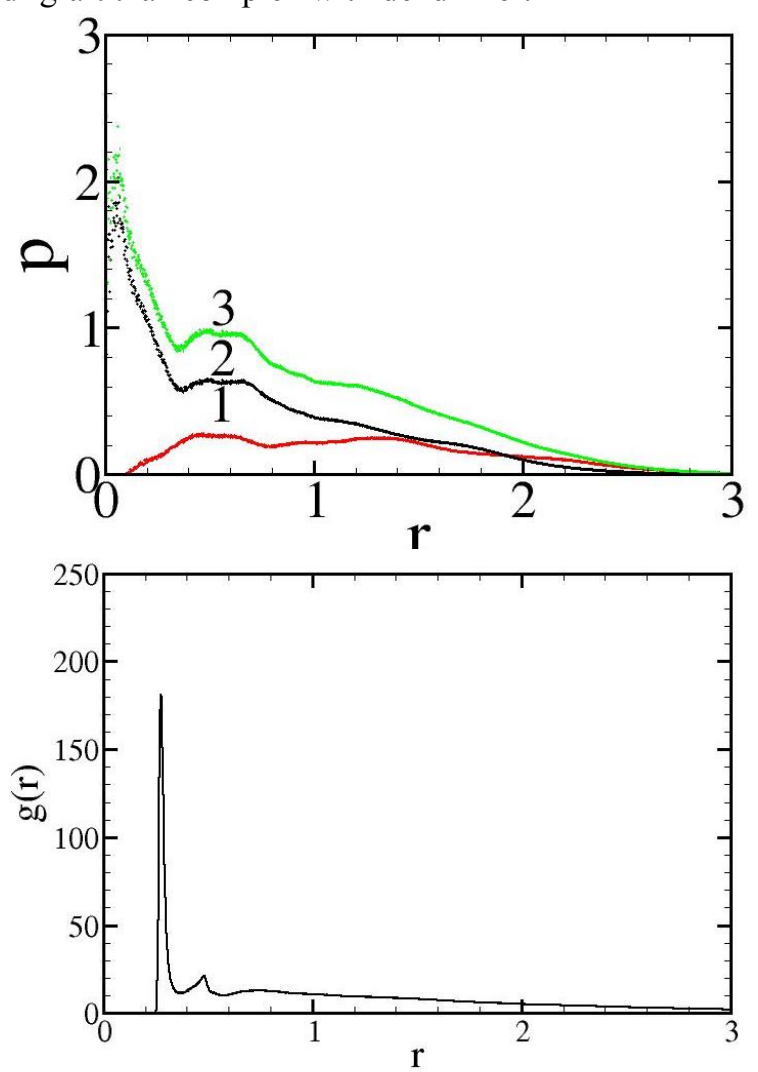

(a)

(b)

Figure 5 a) Radial density) of complex (3), DG2 dendrigraft (2) and peptides (1) and b) Binary function: $\mathrm{NH} 3+$ groups of dendrigraft and $\mathrm{COO}-$ groups of tetrapeptides.
The shape of dendrigraft and dendrimer in both complexes and of the complexes as a whole can be evaluated by ratio of main largest and smallest components ( $\operatorname{Rg} 33 / \operatorname{Rg} 11)$ of equivalent ellipsoid for given system. For dendrigraft DG2 this value is equal to 1,31 and for dendrimer in complex it is equal 1.43, For the complex of DG2 and 16 AEDG peptides it is equal to 1,30 and for complex with dendrimer it is equal 1.39. Thus, the shapes of dendrigraft in complex and of its complex and dendrimer in complex and of its complex are very close to each other. Dendrimer and its complex slightly more anisotropic than dendrigraft and its complex. But the shape in all cases only slightly deviates from spherical shape so it is possible to treat both dendrigraft and dendrimer and their complexes as nearly spherical objects in the rest of the paper and study their radial density distribution functions.

Information about the internal structure of the equilibrium complex could be obtained using radial density distribution function of different subsystem of atoms relatively center of inertia of system $r$. These dependences were calculated using g_rdf function of GROMACS package.

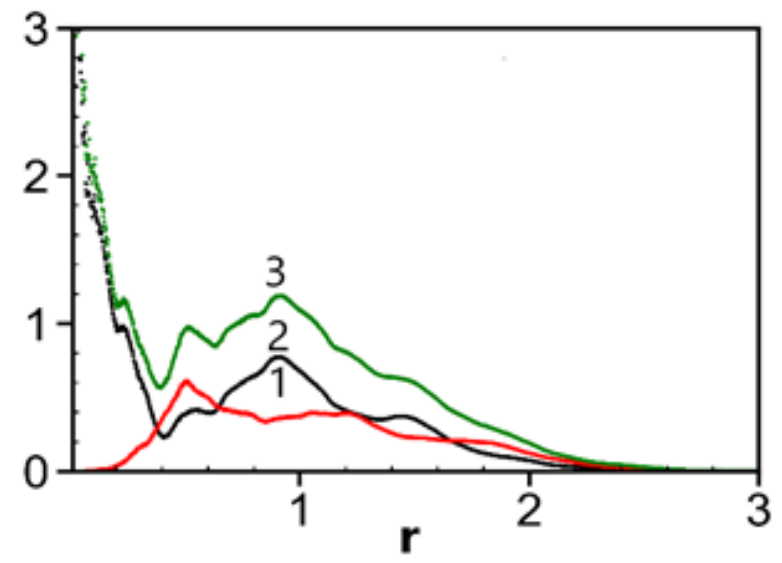

(a)

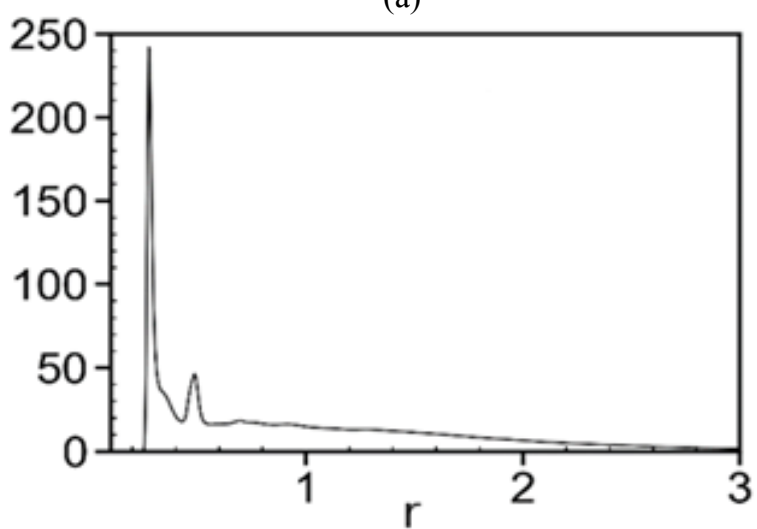

(b)

Figure 6 a) Radial density of complex (3), K2R dendrimer (2), peptides (1), b) Binary function positively charged groups of $\mathrm{K} 2 \mathrm{R}$ and $\mathrm{COO}-$ groups of tetrapeptides. 
Fig.5a and Fig.6a demonstrates that atoms of dendrigraft and dendrimer (curve 2, Fig.5a and Fig.6a) are located mainly in the center of the complex (close to radial distance $r=0$ ). The radial distribution of the dendrigraft density is close to the distributions obtained earlier in separate dendrimers and dendrigrafts of the 2nd generation in water and in their complexes with oppositely charged oligopeptides. At the same time, the density distribution of the new arginine-containing K2R dendrimer is extremely unusual. Earlier, neither for individual lysine dendrimers and dendrigrafts of the 2nd generation in water nor for their complexes with opposite peptides was it possible to obtain such a pronounced minimum of the radial density of distribution of dendrimer atoms in the complex. This result can be explained by the fact that in conventional lysine dendrimers, only terminal lysine amino acid residues are charged, and the inner part of the dendrimer is rather hydrophobic. Therefore, water molecules cannot penetrate deeply into lysine dendrimer. In the new K2R peptide dendrimer, hydrophilic arginine spacers are inserted between all internal branch points of the dendrimer. This leads to an increase in the hydrophilicity of the dendrimer and a greater penetration of water molecules into the dendrimer and a decrease in the density of atoms of the dendrimer in the complex. Peptide atoms (curve 1) could penetrate into dendrimer and there is wide plateau of density for them in both complexes between $\mathrm{r}=0.5 \mathrm{~nm}$ and $1.5 \mathrm{~nm}$ from center of dendrigraft or dendrimer. Due to positive charge of internal arginine spacers in $\mathrm{K} 2 \mathrm{R}$ dendrimer the density of tetrapeptides (curve 1) is greater inside it than in dendrigraft.

The other characteristic of interaction between dendrigraft and peptides in complexes is the pair distribution function between positively charged groups of dendrigraft or dendrimer and negative (COO-) groups of peptide molecules as function of distance between them. Fig. 5b and Fig.6b shows these dependences. It is clearly seen from fig. 5 that there is a sharp peak (curve 1), corresponding to the direct contact (ion pairs) between positively charged groups of dendrigraft or dendrimer and negatively charged COO- groups of in tetrapeptide molecules. Big peak in curve 1 confirms the stable strong electrostatic interactions between dendrigraft or dendrimer and tetrapeptide molecules and their great contribution to stabilization of both complexes. Because this peak is about 1.33 times higher for $\mathrm{K} 2 \mathrm{R}$ dendrimer it has more ion pairs and thus more stable complex.

\section{CONCLUSION}

The process of complex formation of 16 AEDG peptide molecules with lysine dendrigraft or with $\mathrm{K} 2 \mathrm{R}$ dendrimer of similar molecular mass and charge was studied. It was shown that stable dendrigraft-tetrapeptide and dendrimertetrapeptide complexes were formed very quickly. General structure of complex is similar for both systems: dendrimer/dendrigraft atoms stay mainly in center of complex while tetrapeptide atoms are closer to its surface. In this article, it was shown for the first time that the atomic density of a peptide dendrimer with positively charged arginine spacers in a complex with oppositely charged tetrapeptides can have a pronounced minimum. Radial density distribution of AEDG tetrapeptide molecules in dendrigraft and dendrimer complexes also have rather unusual broad plateau range between $\mathrm{r}=0.5$ $\mathrm{nm}$ and $1.8 \mathrm{~nm}$ relatively center of complex. The complexes exist in both cases due to strong electrostatic interactions. In particular there are strong contacts.due to hydrogen bonds (with twice more H-bonds in complex with new dendrimer than in complex with dendrigraft). Ionic pairs between dendrigraft or dendrimer positive charges and COO- groups of tetrapeptide molecules give another important contribution to stability of complexes (with about 1.7 times higher first peak for new dendrimer complex in comparison with dendrigraft complex). Thus, it was obtained that complex of tetrapeptide molecules with dendrimer $\mathrm{K} 2 \mathrm{R}$ is more stable than with dendrigraft of second generation having similar molecular mass and charge. It means that branched polymers with charged internal spacer are better suited to deliver oppositely charged drug molecules. This result can be used to improve the delivery of such drugs.

\section{ACKNOWLEDGMENTS}

The research is carried out using equipment of the shared research facilities of HPC computing resources at Lomonosov Moscow State University. E.I.Fatullaev and I.M.Neelov are supported by RFBR grant 20-53-12036, 20-33-90292.

\section{REFERENCES}

[1] Anisimov V.N., Khavinson V.K., Peptide bioregulation of aging: results and prospects Biogerontology, 2010, 11 (2), 139-149.

[2] Khavinson V. Kh., Malinin V.V. Gerontological aspects of genome peptide regulation, Basel (Switzerland): Karger AG, 2005. - 104 p.

[3] Khavinson V., Diomede F., Mironova E., Linkova N., Trofimova S., Trubiani O., Caputi S., Sijari B., AEDG Peptide Stimulates Gene Expression and Protein Synthesis during Neurogenesis, Molecules,2020, 25, 609.

[4] Denkewalter R.G., Kolc J., Lukasavage W.J. Macromolecular Highly Branched Homogeneous Compound Based on Lysine Units. U.S. Patent US42898721981, 15 September 1981.

[5] Cottet H., Martin M., Papillaud A., Souaid E., Collet H., Commeyras A., Determination of Dendrigraft Poly-L-Lysine Diffusion Coefficients by Taylor Dispersion Analysis, Biomacromolecules,2007, 8, 3235 .

[6]Oukacine F., Romestand B., Goodall D.M., Massiera G., Garrelly L., Cottet H., Study of antibacterial activity by capillary electrophoresis using multiple UV detection points, Anal. Chem., 2012, 84, 3302.

[7] Rossi J.-C., Boiteau L., Collet H., Tsamba B.M., Larcher N., Pascal R., Functionalisation of free amino groups of lysine dendrigraft (DGL) polymers, Tetrahedron Letters, 2012, 53, 2976. 
[8] Cadiere A., Couturaud B., Boismard J., Le Cann P., Gerard A., Mas A., Faye C., Assessment of poly-L -lysine dendrigrafts for virus concentration in water, J. Appl. Microbiol.,2013, 115, 290.

[9] Rao, C.; Tam, J. Synthesis of peptide dendrimer. J. Am. Chem. Soc. 1994, 116, 6975-6976.

[10] Klajnert B., Janiszewska J., Urbanczyk-Lipkowska Z., Bryszewska M., Shcharbin D., Labieniec M. Biological properties of low molecular mass peptide dendrimers. Int. J. Pharm. 2006, 309, 208-217.

[11] Polcyn P. Zielinska P., Zimnicka, M., Troc A., Kalicki P., Solecka J., Laskowska A., UrbanczykLipkowska Z. Novel antimicrobial peptide dendrimers with amphiphilic surface and their interactions with phospholipids - Insights from mass Spectrometry, Molecules 2013, 18, 7120 7144.

[12] Abraham, M.J.; Murtola, T.; Schulz, R.; Pall, S.; Smith, J.C.; Hess, B.; Lindahl, Software X 2015, 1$2,19$.

[13] Lindorff-Larsen, K.; Piana, S.; Palmo, K.; Maragakis, P.; Klepeis, J.L.; Dror, R.O.; Shaw, D.E.

Proteins, 2010, 78, 1950-1958.

[14] Ennari, J., Sundholm, F. Simulation of a PEO based solid polyelectrolyte, comparison of the CMM and the Ewald summation method, Polymer, 2000, 41(6), 2149.

[15] Ennari, J., Sundholm, F. Molecular dynamics simulation of the PEO sulfonic acid anion in water, Computational and Theoretical Polymer Science, 2000, 10(5), 403-410.

[16] Darinsky, A., Lyulin, A., Computer simulations of molecular motion in liquid crystals by the method of Brownian dynamics, Macromolecular Theory and Simulations, 1993, 2(4), 523-530.

[17] M Ennari, J., Sundholm, Molecular dynamics simulation of the structure of PEO based solid polymer electrolytes F. Polymer, 2000, 41(11), 4057-4063.

[18] Neelov, I.M., Binder, K. Brownian dynamics of grafted polymer chains: time dependent properties

Macromolecular Theory and Simulations, 1995, 4(6), 1063-1084

[19]. Neelov, I.M., Binder, K. Brownian dynamics simulation of grafted polymer brushes, Macromolecular Theory and Simulations, 1995, 4(1), 119-136.

[20] Ennari, J., Sundholm, F. Estimation of the ion conductivity of a PEO-based polyelectrolyte system by molecular modeling, Polymer, 2001, 42(19), 8043-8050.

[21] Darinskii, A.A., Gotlib, Yu.Ya., Lyulin, A.V., Computer simulation of local dynamics of a polymer chain in the orienting field of the LC type,

Vysokomolekularnye Soedineniya. Seriya A, 1991, (6), 1211-1220.

[22] Ennari, J., Sundholm, F. Modelling of gas transport properties of polymer electrolytes containing various amounts of water, Polymer, 2004, 45(12), 4171-4179.

[23] Darinskii, A., Gotlib, Yu, Lukyanov, M., Lyulin, A., Computer simulation of the molecular motion in LC and oriented polymers, Application of Scattering Methods to the Dynamics of Polymer Systems, Progr. in Colloid \& Polymer Sci, 1993, 91,13-15.

[24] Ennari, J., Elomaa, M., Sundholm, F. Modeling of water-free and water containing solid polyelectrolytes, Polymer, 2000, 41(3), 985-990.

[25] Falkovich, S.G., Darinskii, A.A. Mechanism of shear deformation of a coiled myosin coil: Computer simulation. Polymer Science - Series A, 2010, 52(6), 662-670.

[26] Gowdy, J., Batchelor, M., Paci, E. Nonexponential Kinetics of Loop Formation in Proteins and Peptides: A Signature of Rugged Free Energy Landscapes? Journal of Physical Chemistry B, 2017, 121(41), 9518-9525.

[27] Gotlib, Y.Y., Balabaev N.K., Darinskii A.A., Investigation of Local Motions In Polymers by the Method of Molecular-Dynamics, Macromolecules,1980 13 (3), 602--608.

[28] Ya. Gotlib, Yu., Darinsky, A.A., Klushin, Properties of kinetic element and local mobility of polymer chains, Acta Polymerica, 1984, 35(2), 124-129.

[29] Lyulin, A.V., Al-Barwani, M.S., Allen, M.P., Allsopp, N.K. Molecular dynamics simulation of main chain liquid crystalline polymers, Macromolecules, 1998, 31(14), 4626-4634.

[30] Neelov, I.M., Borisov, O.V., Binder, K., Stochastic dynamics simulation of grafted polymer brushes under shear deformation, Macromolecular Theory and Simulations, 1998, 7(1), 141-156.

[31] Tarabukina E., Fatullaev E., Krasova A., Sokolova M., Kurlykin M., Neelov I., Tenkovtsev F., Filippov A. Thermoresponsive Molecular Brushes with a Rigid- Chain Aromatic Polyester Backbone and Poly- 2- alkyl- 2- oxazoline Side Chains, s. Int. J. Mol. Sci. 2021, 22, 2265.

[32] Neelov, I.M., Borisov, O.V., Binder, K., Shear deformation of two interpenetrating polymer brushes: Stochastic dynamics simulation, Journal of Chemical Physics, 1998, 108(16), 6973-6988.

[33] Darinskii, A.A., Zarembo, A., Balabaev, N.K., Sundholm, F. Anisotropy of diffusion in a liquid crystalline system of semi-flexible polymer chains

Physical Chemistry Chemical Physics, 2003, 5(11), 2410-2416.

[34]. Ennari, J., Sundholm, F. Simulation of a PEO based solid polyelectrolyte, comparison of the CMM and the Ewald summation method Polymer, 2000, 41(6), 2149.

[35] Okrugin, B.M., Richter, R.P., Leermakers, F.A.M....Borisov, O.V., Zhulina, E.B.,.Structure and properties of polydisperse polyelectrolyte brushes studied by self-consistent field theory, Soft Matter, 2018, 14(30), 6230-6242.

[36] Sheridan, P.F., Adolf, D.B., Lyulin, A.V., Davies, G.R. Computer simulations of hyperbranched 
polymers: The influence of the Wiener index on the intrinsic viscosity and radius of gyration, Journal of Chemical Physics, 2002, 117(16), 7802-7812.

[37] Neelov, I.M., Janaszewska, A., Klajnert, B., ...Turner, A.J., Nalivaeva, N.N. Molecular properties of lysine dendrimers and their interactions with a $\beta$-peptides and neuronal cells, Current Medicinal Chemistry, 2013, 20(1), 134143.

[38] Neelov, I.M., Adolf, D.B., McLeish, T.C.B., Paci, E. Molecular dynamics simulation of dextran extension by constant force in single molecule AFM, Biophysical Journal, 2006, 91(10), 35793588 .

[39] Falkovich, S., Markelov, D., Darinskii, A. Are structural properties of dendrimers sensitive to the symmetry of branching? Computer simulation of lysine dendrimers, Journal of Chemical Physics, 2013, 139(6), 064903.

[40] Neelov, I.M., Markelov, D.A., Falkovich, S.G., ...Okrugin, B.M., Darinskii, A.A. Mathematical simulation of lysine dendrimers: Temperature dependences, Polymer Science - Series C, 2013, 55(1), 154-161).

[41] Neelov, I.M., Adolf, D.B. Brownian dynamics simulation of hyperbranched polymers Under elongational flow, Journal of Physical Chemistry B, 2004, 108(23), 7627-7636.

[42] Neelov, I.M., Adolf, D.B. Brownian dynamics simulations of dendrimers under elongational flow: Bead-rod model with hydrodynamic interactions Macromolecules, 2003, 36(18), 6914-6924.

[43] Mazo, M.A., Shamaev, M.Y., Balabaev, N.K., Darinskii, A.A., Conformational mobility of carbosilane dendrimer: Molecular dynamics simulation

Physical Chemistry Chemical Physics, 2004, 1285-1289.

[44] Neelov, I.M., Adolf, D.B., Lyulin, A.V., Davies, G.R. Brownian dynamics simulation of linear polymers under elongational flow, Journal of Chemical Physics, 2002, 117(8), 4030-4041.

[45] Okrugin, B.M., Leermakers, F.A.M., Borisov, O.V. Structure of asymmetrical peptide dendrimers: Insights given by self-consistent field theory, Polymer, 2017, 125, 292-302.

[46] Okrugin, B., Ilyash, M., Markelov, D., Lysine dendrigraft nanocontainers. Influence of topology. Pharmaceutics, 2018, 10(3), 129

[47] Shavykin, O.V., Leermakers, F.A.M.,Darinskii, A.A. Self-Assembly of Lysine-Based Dendritic Surfactants Modeled by the Self-Consistent Field Approach, Langmuir, 2018, 34(4), 1613-1626.

[48] Shavykin, O.V., Mikhailov, I.V., Darinskii, A.A. Leermakers, F.A.M. Effect of an asymmetry of branching on structural characteristics of dendrimers revealed by simulations, Polymer, 2018, 146, 256-266

[49] Shavykin, O.V., Borisov, O.V., Darinskii, A.A., Leermakers, F.A.M SCF Theory of Uniformly
Charged Dendrimers, Macromolecules, 2020, 53(17), стр. 7298.

[50] Shavykin, O.V., Darinskii, A.A. Is the manifestation of the local dynamics in the spin-lattice NMR relaxation in dendrimers sensitive to excluded volume interactions? Physical Chemistry Chemical Physics, 2016, 18(35), 24307-24317.

[51] Sheveleva, N.N., Markelov, D.A., Vovk, M.A., ... Lähderanta, E., Lysine-based dendrimer with double arginine residues, RSC Advances, 2019, 9(31), 18018.

[52] Neelov, I.M., Shavykin, O.V., Ilyash, M.Y., ...Darinskii, A.A., Leermakers, F.A.M. Application of high-performance computing for comparison of two highly branched lysine molecules of different topology,

Supercomputing Frontiers \& Innovations, 2018, 5(3), 60.

[53] Sheveleva, N.N., Markelov, D.A., Vovk, M.A.,..Lahderanta, E.Stable deuterium labeling of histidine-rich lysine-based dendrimers, Molecules, 2019, 24(13), 2481.

[54] Bezrodnyi, V.V., Shavykin, O.V., Mikhtaniuk, S.E., ... Markelov, D.A.Molecular Dynamics and SpinLattice NMR Relaxation in $\alpha$ - and $\varepsilon$-Polylysine, Applied Magnetic Resonance, 2020, 51(12), 16691679.

[55] Gorzkiewicz, M., Kopeć, O., Janaszewska, A., ... Klajnert-Maculewicz,B.Poly(Lysine) dendrimers form complexes with siRNA and provide its effcient uptake by myeloid cells, International Journal of Molecular Sciences, 2020, 21(9), 3138.

[56] Sheveleva, N.N., Markelov, D.A., Vovk, M.A.,..Lähderanta, E., NMR studies of excluded volume interactions in peptide dendrimers, Scientific Reports, 2018, 8(1), 8916.

[57] Gorzkiewicz, M., Konopka, M., Janaszewska, A., ..., Klajnert-Maculewicz, B.Application of new lysinebased peptide dendrimers D3K2 and D3G2 for gene delivery: Specific cytotoxicity to cancer cells and transfection in vitro, Bioorganic Chemistry, 2020, 95, 103504.

[58] Bezrodnyi, V.V., Fatullaev, E.I., Mikhtaniuk, S.E., Tarasenko, I.I., Interaction of lysine dendrimer with 8 and 16 molecules of EDR peptide, International Journal of Biology and Biomedical Engineering, 2020, 14, стр. 1-6.

[69] Bezrodnyi, V.V., Shavykin, O.V., Mikhtaniuk, S.E., ...Sheveleva, N.N., Markelov, D.A. Why the orientational mobility in arginine and lysine spacers of peptide dendrimers designed for gene delivery is different? International Journal of Molecular Sciences, 2020, 21(24), 1-22, 9749.

[60]. Mikhtaniuk, S.E., Bezrodnyi, V.V., Shavykin, O.V.,.Penkova, A.V., Markelov, D.A. Comparison of structure and local dynamics of two peptide dendrimers with the same backbone, Polymers, 2020, 12(8), 1657.

[61] Bezrodnyi, V.V., Mikhtaniuk, S.E.,, Shavykin, O.V., Sheveleva N.N., Markelov, D.A. Size and Structure of Empty and Filled Nanocontainer Based on 
Peptide Dendrimer with Histidine Spacers at Different pH, Molecules, 2021, 26 (21), 6552.

[62] Markelov, D.A., Falkovich, S.G.,..Ingman, P., Darinskii, A.A. Molecular dynamics simulation of spin-lattice NMR relaxation in poly-L-lysine dendrimers:, PCCP, 2015, 17(5), 3214-3226.

[63] Khamidova, D.N., Bezrodnyi, V.V., Popova, A.V., ...Popova, E.V. Molecular dynamics simulation of complexes of lysine dendrimer and dendrigraft with AENG tetrapeptide International Journal of Biology and Biomedical Engineering, 2018, 12, 45-58

[64] Neelov, I., Popova, E., Khamidova, D., Komilov, F. Interaction of lysine dendrimers of $2 \mathrm{nd}$ and $3 \mathrm{rd}$ generation with stack of amyloid peptides. Molecular dynamics simulation, International Journal of Biology and Biomedical Engineering, 2017, 11, 95-100.

[65] Afshin Poorkhanalikoudehi, Karl-Heinz Zimmermann, Cellular Automaton for Kidney Branching Morphogenesis, WSEAS Transactions on Biology and Biomedicine, vol. 18, 2021, pp. 170-182.

[66] Nigel Aylward, A Computational Study of a Prebiotic Synthesis of L-Threonine, WSEAS Transactions on Biology and Biomedicine, Volume 16, 2019, pp. 138-146

[67] Khamidova, D.N., Bezrodnyi, V.V., Popova, A.V., ...Popova, E.V Molecular dynamics simulation of complexes of lysine dendrimer and dendrigraft with AENG tetrapeptide. International Journal of Biology and Biomedical Engineering, 2018, 12, 717.

[68] I.M. Neelov, E.V Popova, Interaction of lysine dendrimer and Semax peptides. Molecular dynamics simulation. Int. J. Biology\&Biomedicine., 2017, 2, 6-12.

[69] Ilyash, M.Yu., Bezrodnyi, V.V., Fatullaev E.I., ..Tarasenko, I.I., Computer simulation of complexation of lysine dendrigraft of second generation with DS dipeptide molecules International Journal of Biology and Biomedical Engineering, 2019, 13, 63-69.

[70] Neelov, I., Popova, E., Khamidova, D., Tarasenko,Interaction of lysine dendrimers with therapeutic peptides. Molecular dynamics simulation. I.International Journal of Biology and Biomedical Engineering, 2017, 11, 194-203.

[71] Neelov, I., Khamidova, D., Bezrodnyi, V., Mikhtaniuk, S Molecular dynamics simulation of interaction of lysine dendrigraft of 2 nd generation with stack of amyloid peptides. International Journal of Biology and Biomedical Engineering, 2019, 13, 26-31.

\section{Contribution of Individual Authors to the Creation of a Scientific Article (Ghostwriting Policy)}

Emil Fatullaev carried out the simulations and analysis of data for dendrigraft and its complex with peptide molecules. Valerii Bezrodnyii carried out the simulations and analysis of data for dendrimer and its complex with peptide molecules. Igor Neelov is the supervisor of both graduate students. He took part in this study during all stages of this work.

Creative Commons Attribution License 4.0 (Attribution 4.0 International, CC BY 4.0)

This article is published under the terms of the Creative Commons Attribution License 4.0

https://creativecommons.org/licenses/by/4.0/deed.en_US 